\section{Enteritis lúpica como manifestación inicial de lupus eritematoso sistémico. Caso clínico}

\author{
MANUEL BARRERA O. ${ }^{a}$, RODRIGO BARRERA M. ${ }^{a}$, \\ MATÍAS DE LA RIVERA V.a, JAVIER VELA U.a, \\ GUSTAVO MÖNCKEBERG F.
}

\section{Lupus enteritis as initial manifestation of systemic lupus erythematosus. Report of one case}

Although gastrointestinal symptoms are not rare in Systemic lupus erythematosus, enteritis is an atypical manifestation of the disease. We report a 54 year-old woman who presented acute symptoms of diarrhea, fever and abdominal pain, receiving empiric antibiotic therapy for bacterial enteritis with no response. Computed tomography showed diffuse small intestine inflammation and serositis. Antinuclear antibodies, anti-Ro and anti-La were positive on blood tests. A lupic enteropathy was diagnosed and steroid treatment was initiated, with subsequent clinical improvement.

(Rev Med Chile 2017; 145: 1349-1352)

Key words: Autoimmune Diseases; Enteritis; Glucocorticoids; Lupus Erythematosus, Systemic.

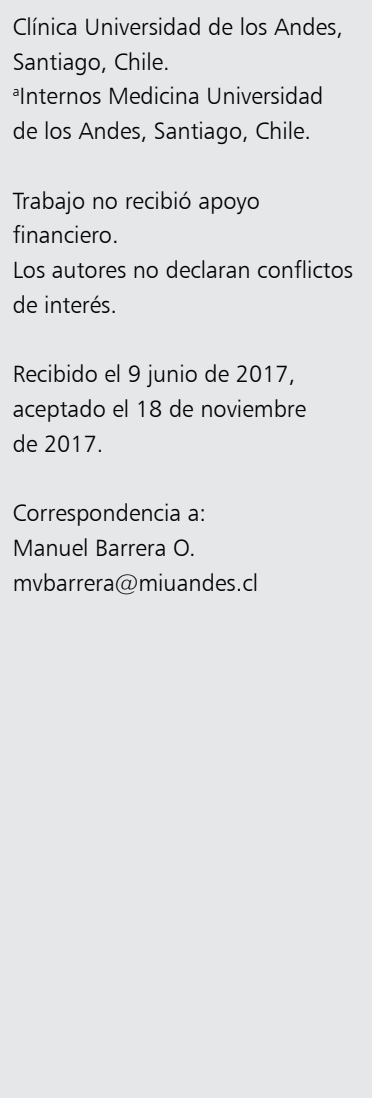

Clínica Universidad de los Andes,

Santiago, Chile.

Internos Medicina Universidad

Trabajo no recibió apoyo

financiero.

Los autores no declaran conflictos

aceptado el 18 de noviembre

mvbarrera@miuandes.cl
E 1 lupus eritematoso sistémico (LES) es una enfermedad inflamatoria autoinmune sistémica de curso crónico, asociada a una elevada morbimortalidad y gran variedad de presentaciones clínicas. El debut de esta enfermedad suele estar dado por la presencia de síntomas cutáneos $(73 \%)$ y musculoesqueléticos $(67 \%)$. Menos frecuente es que estos pacientes debuten con manifestaciones digestivas (18\%) como dolor abdominal agudo, hepatitis autoinmune, pancreatitis aguda, disfagia, enteritis lúpica, entre otras ${ }^{1}$.

La enteritis lúpica corresponde a la inflamación de la pared intestinal, provocada por el depósito local de complejos inmunes, con activación del complemento y la presencia de vasculitis. La tomografía axial computada (TAC) es la herramienta más útil para diagnosticar esta patología, habiendo tres signos radiológicos orientadores: engrosamiento de la pared intes- tinal con dilatación de los segmentos comprometidos, también conocido como "signo de tiro al blanco" o "target", ingurgitación de los vasos mesentéricos o "signo de la peineta" y atenuación incrementada de la grasa mesentérica ${ }^{2}$. El estudio histológico no ha demostrado ser de utilidad como herramienta diagnóstica en primera instancia. Se reserva principalmente para descartar otras causas de cuadros similares cuando existe alta duda diagnóstica o el estudio inicial no fue concluyente.

Algunos pacientes pueden desarrollar, durante su evolución, complicaciones graves: infarto, obstrucción y perforación intestinal. Se han descrito algunos factores predictores de mal pronóstico: leucopenia, hipoalbuminemia y amilasa sérica elevada ${ }^{3}$.

El presente artículo tiene como objetivo presentar la enteritis lúpica como una forma infrecuente de presentación de LES, a propósito 
del caso de una paciente de 54 años que debutó con dicha manifestación. Además, identificar sus principales características clínicas, de laboratorio e imágenes. Finalmente revisar el manejo terapéutico más aceptado y su pronóstico a futuro.

\section{Caso clínico}

Se presenta el caso de una paciente mujer de 54 años, con antecedentes de enfermedad celiaca, vitíligo y lupus cutáneo crónico diagnosticado con biopsia de piel 10 años antes. Consulta en el Servicio de Urgencias por un cuadro diarreico agudo de una semana de evolución, asociado a fiebre y dolor abdominal cólico difuso, náuseas y vómitos. No había presencia de sangre o pus en las deposiciones.

Entre los exámenes realizados destaca: leucocitos de $4.600 \mathrm{u} / \mathrm{L}$ con linfopenia de un $9 \%$ y recuento absoluto de $414 \mathrm{u} / \mathrm{L}$, Proteína C Reactiva de $255 \mathrm{mg} / \mathrm{L}$ (normal: 0-5 mg/L), lipasa elevada de 280 U/L (normal: 0,1-66 U/L), leucocitos fecales positivos, hipoalbuminemia de $2,8 \mathrm{~g} / \mathrm{dL}$ (normal: $3,5-5,2 \mathrm{~g} / \mathrm{dL}$ ) y proteinuria de $800 \mathrm{mg}$ en $24 \mathrm{~h}$ (normal: $40-150 \mathrm{mg}$ ) con sedimento de orina no inflamatorio. Se realizó estudio de Clostridium difficile mediante reacción en cadena de polimerasa (RCP) y filmarray gastrointestinal mediante la misma técnica, resultando ambos negativos. $\mathrm{La}$ TAC de abdomen mostró asas de yeyuno e íleon con engrosamiento e hipercaptación parietal, de aspecto inflamatorio, con leve engrosamiento parietal de colon a nivel de ángulo esplénico, aumento de atenuación del tejido adiposo mesentérico y líquido libre de leve cuantía en cavidad pélvica. Estos hallazgos fueron interpretados como una enterocolitis bacteriana (Figura 1). Al ingreso se inició tratamiento antibiótico empírico con ciprofloxacino $200 \mathrm{mg}$ cada $12 \mathrm{~h}+$ metronidazol $500 \mathrm{mg} \mathrm{c} / 8 \mathrm{~h}$ endovenosos.

Al cuarto día de evolución había persistencia de la fiebre, diarrea y elevación de reactantes de fase aguda. Se decide aumentar cobertura antibiótica con piperacilina/tazobactam $4 \mathrm{~g} / 0,5 \mathrm{~g}$ cada $8 \mathrm{~h}$ y control de imágenes con nuevo TAC de abdomen y pelvis, el cual mostró derrame pleural bilateral, ascitis y progresión de la enterocolitis con respecto a la imagen anterior (Figura 2). Dados los antecedentes de la paciente, el curso clínico refractario al uso de antibióticos de amplio espectro, la ausencia de demostración microbiológica de infección y la aparición de serositis; se decide realizar estudio de autoinmunidad y se inicia prueba terapéutica con metilprednisolona $125 \mathrm{mg}$ endovenoso por 3 días, con respuesta clínica rápida y satisfactoria. Dentro de los resultados de exámenes destacan: ANA (+) 1/80 patrón moteado, anti Ro y anti La (+), C3 53,2 mg/dL (normal: 70-180 mg/dL), C4 $19,7 \mathrm{mg} / \mathrm{dL}$ (normal: $16-45 \mathrm{mg} / \mathrm{dL}$ ). El resto de los marcadores fueron negativos (anti-DNA y estudio síndrome anti fosfolípido en sus 3 técnicas).

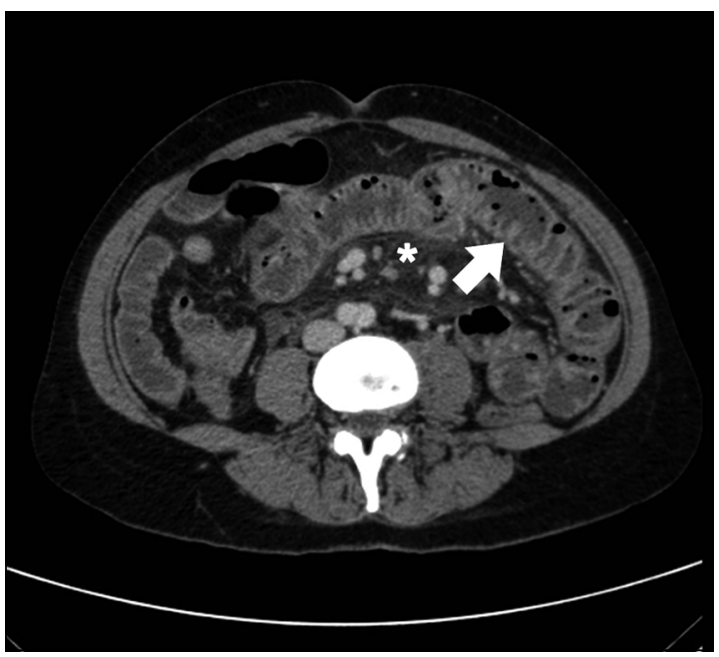

Figura 1. Flecha indica asas intestinales engrosadas e hipercaptación parietal. El asterisco señala el aumento de la atenuación del tejido adiposo mesentérico.

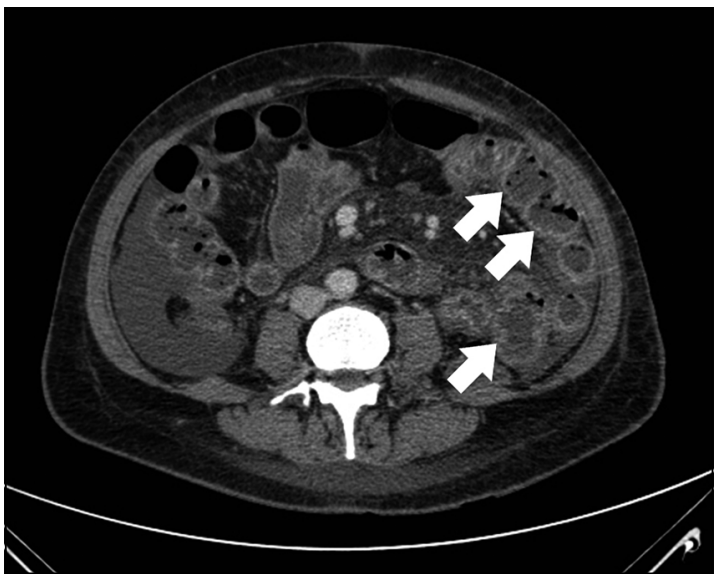

Figura 2. Flechas señalan la progresión del compromiso de las asas intestinales. 
Con estos elementos se planteó el diagnóstico de enteritis lúpica, en contexto de un lupus eritematoso sistémico activo. Se continúa terapia con prednisona en dosis de $1 \mathrm{mg} / \mathrm{Kg} /$ día vía oral; con desaparición de la fiebre, dolor y diarrea, además de la normalización rápida de los parámetros inflamatorios.

\section{Discusión}

El LES es una entidad clínica cuyo modo de presentación, evolución y pronóstico es muy heterogéneo; con una gran variedad de manifestaciones clínicas, las que muchas veces se van sumando en el tiempo, alternando períodos de actividad y quiescencia y sin que exista un cuadro patognomónico. Todo lo anterior explica la dificultad en el diagnóstico, lo que muchas veces impide llegar en etapas precoces de la enfermedad. Se han elaborado múltiples criterios de clasificación, pensados para hablar con una nomenclatura común y uniforme, pero que no necesariamente resultan útiles al momento del diagnóstico individual. Los criterios ACR del año 1982, han sido recientemente revisados y reemplazados por los criterios SLICC 2012 ${ }^{4}$. En el caso particular expuesto en el artículo, la paciente cumple con más de 4 criterios requeridos para la clasificación: lupus cutáneo crónico, trastornos hematológicos, serositis, proteinuria y ANA (+).

Dentro de las manifestaciones del LES, la enteritis lúpica requiere de un alto índice de sospecha incluso en pacientes diagnosticados con LES previamente, ya que su aparición es poco frecuente.

Se ha descrito que su prevalencia alcanza el 0,2-2\% en el total de pacientes con LES, y $45-79 \%$ en pacientes con LES que se presentan con dolor abdominal ${ }^{5}$.

Dentro de las manifestaciones más frecuentes se encuentra el dolor abdominal (97\%) seguido de vómitos $(42 \%)$ y diarrea (32\%). Estos síntomas se pueden acompañar de hallazgos de laboratorio inespecíficos como anemia, leucopenia, linfopenia, trombocitopenia y en algunos casos proteinuria $^{6}$.

Como experiencia local, existe un caso reportado en el Hospital San Borja Arriarán de apendicitis secundario a una vasculitis lúpica, cuya presentación clínica fue de dolor abdominal en fosa iliaca derecha e irritación peritoneal, asociado a compromiso cutáneo y articular7 .

El examen más útil para el diagnóstico es la TAC de abdomen. Dentro de los hallazgos clásicos se incluyen engrosamiento difuso de la pared intestinal, dilatación de asas intestinales, ensanchamiento e hipervascularización esplácnica, atenuación incrementada de la grasa mesentérica $y$ ascitis ${ }^{6-8}$.

Sin embargo, se ha visto que en casos donde la sospecha de enteritis lúpica es alta, la ecografía podría ser una herramienta útil para detectar edema intestinal precoz'.

Los hallazgos característicos en esta paciente que permitieron orientar el diagnóstico hacia esta patología fueron la presencia de enteritis y atenuación de la grasa mesentérica, a la cual se agregó serositis (ascitis y derrame pleural).

Se ha visto que el estudio con colonoscopía y biopsia no aporta mayor utilidad al diagnóstico definitivo ya que los vasos sanguíneos afectados no se logran alcanzar mediante este examen. Además, los hallazgos son inespecíficos, mostrando un proceso inflamatorio a nivel de submucosa, con necrosis fibrinoide y leucocitoclástica de la pared vascular. Es por esta razón que en nuestro caso no se consideró el estudio histológico como opción ${ }^{10}$.

En cuanto al manejo, actualmente no hay un consenso establecido sobre la mejor elección terapéutica, debido a la falta de ensayos clínicos sobre este tema. El tratamiento habitual e inicial es en general con metilprednisolona o prednisona oral en dosis altas. Para casos corticoide-resistente se puede utilizar ciclofosfamida o micofenolato, teniendo este último un mejor perfil de seguri$\mathrm{dad}^{11}$. En casos refractarios a ciclofosfamida $\mathrm{u}$ otros inmunosupresores, el uso de tacrolimus ha mostrado ser eficaz en reporte de $\operatorname{casos}^{12}$.

El pronóstico en general es bueno y la recurrencia poco frecuente, sin embargo, la presencia de los siguientes factores: afectación colónica, del tracto urinario ${ }^{5}$ y el grosor de la pared intestinal $>8 \mathrm{~mm}^{3}$, podrían asociarse a una mayor tasa de recurrencia.

En conclusión, debemos considerar la enteritis lúpica como posible manifestación digestiva inicial en pacientes con LES. Su diagnóstico requiere de un alto índice de sospecha, siendo la TAC uno de los pilares fundamentales para el diagnóstico. El manejo más aceptado es con el uso de corticoides o inmunomoduladores, logrando una buena respuesta y pronóstico a largo plazo. 


\section{Referencias}

1. Von Feldt JM. Systemic lupus erythematosus. Recognizing its various presentations. Postgrad Med 1995; 97 (4): 79, 83, 86 passim.

2. Smith LW, Petri M. Lupus enteritis: an uncommon manifestation of systemic lupus erythematosus. J Clin Rheumatol Pract Rep Rheum Musculoskelet Dis 2013; 19 (2): 84-6.

3. Yuan S, Ye Y, Chen D, Qiu Q, Zhan Z, Lian F, et al. Lupus mesenteric vasculitis: clinical features and associated factors for the recurrence and prognosis of disease. Semin Arthritis Rheum 2014; 43 (6): 759-66.

4. Petri M, Orbai A-M, Alarcón GS, Gordon C, Merrill JT, Fortin PR, et al. Derivation and validation of the Systemic Lupus International Collaborating Clinics classification criteria for systemic lupus erythematosus. Arthritis Rheum 2012; 64 (8): 2677-86.

5. Lee HA, Shim HG, Seo YH, Choi SJ, Lee BJ, Lee YH, et al. Panenteritis as an Initial Presentation of Systemic Lupus Erythematosus. Korean J Gastroenterol Taehan Sohwagi Hakhoe Chi 2016; 67 (2): 107-11.

6. Janssens P, Arnaud L, Galicier L, Mathian A, Hie M, Sene D, et al. Lupus enteritis: from clinical findings to therapeutic management. Orphanet J Rare Dis 2013; 8: 67.

7. Barrera A, Bannura C, Corredoira Y, García A. Apendicitis aguda secundaria a una vasculitis lúpica. Revista Chilena de Cirugía 2002; 54 (5): 529-31.

8. Yagita M, Tsujimoto K, Yagita M, Fujita M. Atypical Presenting Symptoms of Acute Onset Systemic Lupus Erythematosus with Enteritis and Cystitis. Case Rep Med 2016; 2016: 8579812.

9. Demiselle J, Sayegh J, Cousin M, Olivier A, Augusto J-F. An Unusual Cause of Abdominal Pain: Lupus Enteritis. Am J Med 2016; 129 (5): e11-12.

10. Peña R, Almanza E, Vargas E, Zaragoza G, Espinosa P, Castañeda R, Chávez L. Vasculitis gastrointestinal en una adolescente con lupus eritematoso sistémico y síndrome anti fosfolípido catastrófico. Endoscopía 2016; 28 (4): 174-77.

11. Al Balushi F, Humby F, Mahto A, Kelly C, Jawad A. Mycophenolate mofetil inducing remission of lupus enteritis. Lupus 2012; 21 (5): 556-8.

12. Shirai T, Hirabayashi Y, Watanabe R, Tajima Y, Fujii H, Takasawa N, et al. The use of tacrolimus for recurrent lupus enteritis: a case report. J Med Case Reports 2010; 4: 150. 